\title{
A GENERALIZATION OF THE SIERPIŃSKI THEOREM
}

\author{
J. J. DIJKSTRA
}

\begin{abstract}
Sierpiński's theorem admits the following generalization. Let $n$ be a nonnegative integer and $X$ a compact Hausdorff space. If $\left\{F_{i} \mid i \in \mathbf{N}\right\}$ is a countable closed covering of $X$ such that $\operatorname{dim}\left(F_{i} \cap F_{j}\right)<n$ for distinct $i$ and $j$ in $\mathbf{N}$, then every continuous mapping from $F_{1}$ into the $n$-sphere $S^{n}$ is extendable over $X$.
\end{abstract}

Consider the following well-known theorem (see Engelking [3, p. 440] or, for sets in $\mathbf{R}^{n}$, Sierpiński [4]).

THEOREM 1. Let $X$ be a continuum (i.e. a compact, connected Hausdorff space). If $\left\{F_{i} \mid i \in \mathbf{N}\right\}$ is a pairwise disjoint, closed covering of $X$ then $F_{i}=X$ for some $i \in \mathbf{N}$.

The aim of this paper is to prove the following assertion, where dim stands for the covering dimension.

THEOREM 2. Let $n$ be a nonnegative integer and $X$ a compact Hausdorff space. If $\left\{F_{i} \mid i \in \mathbf{N}\right\}$ is a closed covering of $X$ such that $\operatorname{dim}\left(F_{i} \cap F_{j}\right)<n$ for every $i$ and $j$ with $i \neq j$, then every continuous mapping from $F_{1}$ into the $n$-sphere $S^{n}$ is continuously extendable over $X$.

The reader is encouraged to verify that Theorem 1 follows easily from Theorem 2 if one substitutes $n=0$. We first prove the theorem for metric spaces.

LEMMA 3. Theorem 2 is valid in the class of metric spaces.

ProOF. We shall work with the following induction hypothesis for $n=0,1$, $2, \ldots$

Let $X$ be a compact metric space and $M$ an absolute retract (AR). If $\left\{F_{i} \mid i \in \mathbf{N}\right\}$ is a closed covering of $X$ such that $\operatorname{dim}\left(F_{i} \cap F_{j}\right)<n$ for every $i$ and $j$ with $i \neq j$, then every continuous $f: F_{1} \rightarrow S^{n} \times M$ is extendable over $X$.

Consider the case $n=0$, where we have $S^{n}$ is the discrete doubleton $\{-1,1\}$ and $\left\{F_{i} \mid i \in \mathbf{N}\right\}$ is pairwise disjoint. Assume that the closed set $A=$ $f^{-1}(\{-1\} \times M) \subset F_{1}$ is nonempty. Let $\tilde{X}$ be the space we obtain from $X$ by identifying $A$ to a single point $a$, let $q: X \rightarrow \tilde{X}$ be the decomposition map and let $C$ be the component of $a$ in $\tilde{X}$. $C$ is a continuum with the pairwise disjoint, closed covering $\{\{a\}, B \cap C\} \cup\left\{F_{i} \cap C \mid i \geq 2\right\}$, where $B=f^{-1}(\{1\} \times M)$. According to Theorem 1 we have $C=\{a\}$. Since $X$ is a compact Hausdorff space there is a clopen subset $O$ of $\tilde{X}$ with $a \in O$ and $O \cap B=\emptyset$. Because $M$ is an AR we can find

Received by the editors May 20, 1983 and, in revised form, August 8, 1983.

1980 Mathematics Subject Classification. Primary 54F60.

Key words and phrases. Sierpiński's theorem, covering dimension, $n$-sphere.

(C) 1984 American Mathematical Society $0002-9939 / 84 \$ 1.00+\$ .25$ per page 
continuous mappings $g_{1}: q^{-1}(O) \rightarrow\{-1\} \times M$ and $g_{2}: X \backslash q^{-1}(O) \rightarrow\{1\} \times M$ such that $g_{1}|A=f| A$ and $g_{2}|B=f| B$. Then $g_{1} \cup g_{2}: X \rightarrow S^{0} \times M$ is as required.

Assume now that the induction hypothesis holds for $n$. Let $\left\{F_{i} \mid i \in \mathbf{N}\right\}$ be a closed covering of $X$ such that $\operatorname{dim}\left(F_{i} \cap F_{j}\right) \leq n$ for $i \neq j$, and let $f: F_{1} \rightarrow S^{n+1} \times M$ be continuous. According to the countable sum theorem (Engelking $[\mathbf{2}, 3.1 .8]$ ) the set $B=\bigcup\left\{F_{i} \cap F_{j} \mid i, j \in \mathbf{N}\right.$ with $\left.i \neq j\right\}$ has covering dimension $\leq n$. Let $x_{1}$ and $x_{2}$ be two distinct points in $S^{n+1}$ and note that $S^{n+1} \backslash\left\{x_{1}, x_{2}\right\}$ is homeomorphic to $S^{n} \times \mathbf{R}$. Using the separation theorem (Engelking $\left.[2,4.1 .13]\right)$ we find a closed covering $\left\{H_{1}, H_{2}\right\}$ of $X$ such that $H_{j} \cap f^{-1}\left(\left\{x_{j}\right\} \times M\right)=\emptyset$ for $j=1,2$, and $\operatorname{dim}\left(H_{1} \cap H_{2} \cap B\right)<n$.

Consider the compact space $X^{\prime}=H_{1} \cap H_{2}$ and its closed covering $\left\{F_{i} \cap X^{\prime} \mid i \in\right.$ N\}. Obviously, we have

$$
\operatorname{dim}\left(F_{i} \cap F_{j} \cap X^{\prime}\right) \leq \operatorname{dim}\left(B \cap X^{\prime}\right)<n \quad \text { for } i \neq j .
$$

Observe that $f \mid F_{1} \cap X^{\prime}$ is a continuous mapping into $\left(S^{n+1} \backslash\left\{x_{1}, x_{2}\right\}\right) \times M$, which space is homeomorphic to $S^{n} \times \mathbf{R} \times M$. Since $\mathbf{R} \times M$ is, as a product of AR's, itself an AR we may apply the induction hypothesis to find a continuous

$$
g: X^{\prime} \rightarrow\left(S^{n+1} \backslash\left\{x_{1}, x_{2}\right\}\right) \times M
$$

with $g\left|F_{1} \cap X^{\prime}=f\right| F_{1} \cap X^{\prime}$. Observing that $S^{n+1} \backslash\left\{x_{j}\right\}$ is homeomorphic to $\mathbf{R}^{n+1}$ select continuous functions

$$
h_{j}: H_{j} \rightarrow\left(S^{n+1} \backslash\left\{x_{j}\right\}\right) \times M, \quad j=1,2,
$$

with $h_{j} \mid X^{\prime}=g$ and $h_{j}\left|H_{j} \cap F_{1}=f\right| H_{j} \cap F_{1}$. Then $h=h_{1} \cup h_{2}$ is a continuous mapping from $X$ into $S^{n+1} \times M$ which extends $f$ and the lemma is proved.

Notations. Let $d$ be a pseudo-metric on a space $X$. For $\varepsilon>0$ and $x \in X$ let $S_{\varepsilon}^{d}(x)=\{y \in X \mid d(y, x)<\varepsilon\}$ be the $\varepsilon$-ball with respect to $d$. $\tau_{d}$ denotes that topology for which $\left\{S_{\varepsilon}^{d}(x) \mid x \in X\right.$ and $\left.\varepsilon>0\right\}$ is an open basis. $d$ is called an admissible metric on $X$ if $\tau_{d}$ is contained in the topology of $X$. Let $\mathcal{U}=\left\{U_{i} \mid\right.$ $i=1, \ldots, k\}$ be a finite collection of subsets of $X . \operatorname{mesh}_{d} \mathcal{U}$ is the maximum of the $d$-diameters of the elements of $\mathcal{U}$. If every point of $X$ is contained in at most $n(\geq 0)$ members of $\mathcal{U}$ then we denote this by ord $\mathcal{U} \leq n . \mathcal{V}=\left\{V_{i} \mid i=1, \ldots, k\right\}$ is called a shrinking of $\mathcal{U}$ if for every $i \leq k, V_{i} \subset U_{i}$.

LEMMA 4. Let $n$ be a nonnegative integer. Suppose that $X$ is a normal space and $F$ a closed subset with $\operatorname{dim}(F)<n$. If $\mathcal{U}=\left\{U_{i} \mid i=1, \ldots, k\right\}$ is a finite open covering of $F$ in $X$ then there is an admissible pseudo-metric $d$ on $X$ and a shrinking $\mathcal{V}=\left\{V_{i} \mid i=1, \ldots, k\right\}$ of $\mathcal{U}$ such that $\mathcal{V} \subset \tau_{d}$, ord $\mathcal{V} \leq n$ and $F \subset \cup \mathcal{V}$.

Proof. Let $\mathcal{U}=\left\{U_{i} \mid i=1, \ldots, k\right\}$ be an open covering of $F$ in $X$. Consider $\mathcal{U}_{F}=\left\{U_{i} \cap F \mid i=1, \ldots, k\right\}$ and select an $F$-open shrinking $\mathcal{V}=\left\{V_{i} \mid i=1, \ldots, k\right\}$ of $\mathcal{U}_{F}$ with $\cup \mathcal{V}=F$ and ord $\mathcal{V} \leq n$. Since $F$ is a normal space we may assume that the $V_{i}$ 's are cozero-sets in $F$ such that the closure of $V_{i}$ is contained in $U_{i}$. The set $F$ is closed in the normal space and hence there are cozero-sets $\hat{V}_{i}$ in $X$ such that $\hat{V}_{i} \cap F=V_{i}$. Moreover, since $\operatorname{Cl}\left(V_{i}\right) \subset U_{i}$ we may assume that $\hat{V}_{i} \subset U_{i}$. Using real valued mappings that correspond with the cozero-sets $\hat{V}_{1}, \ldots, \hat{V}_{k}$ we construct an admissible pseudo-metric $d$ on $X$ such that $\hat{V}_{i} \in \tau_{d}$ for $i \leq k$. Define now for every $U \in\left\{O \cap F \mid O \in \tau_{d}\right\}$ the set

$$
U^{\prime}=\{x \in X \mid d(x, U)<d(x, F \backslash U)\} \in \tau_{d},
$$


where $d(x, \emptyset)=\infty$. It is easily verified that $U^{\prime} \cap F=U$ and $U^{\prime} \cap V^{\prime}=(U \cap V)^{\prime}$. This implies that $\operatorname{ord}\left\{\left(V_{i}\right)^{\prime} \mid i=1, \ldots, k\right\} \leq n$ and hence that $\left\{\hat{V}_{i} \cap\left(V_{i}\right)^{\prime} \mid i=1, \ldots, k\right\}$ meets the requirements of this lemma.

We have now concluded the preliminaries and shall prove the theorem.

Proof of TheOREM 2. Let $f$ be a continuous function from $F_{1}$ into $S^{n}$. We construct (inductively) a sequence $\rho_{1}, \rho_{2}, \rho_{3}, \ldots$ of admissible pseudo-metrics on $X$. Let $S^{n}$ be represented by the boundary $\partial I^{n+1}$ of the cube $I^{n+1}$ and let $\bar{f}: X \rightarrow I^{n+1}$ be a continuous extension of $f$. Take for $\rho_{1}$ the preimage under $\bar{f}$ of a metric that corresponds with the euclidean topology on $I^{n+1}$ and that is bounded by 1 . Suppose that we have constructed $\rho_{m}$. Let $\alpha=(i, j) \in A=\{(k, l) \mid k, l \in \mathbf{N}$ with $k \neq l\}$. With Lemma 4 we can find an admissible pseudo-metric $d_{\alpha}$ on $X$ and a finite covering $\mathcal{V}$ of $F_{i} \cap F_{j}$ such that $\mathcal{V} \subset \tau_{d_{\alpha}}$, ord $\mathcal{V} \leq n$ and $\operatorname{mesh}_{\rho_{m}} \mathcal{V}<1 / m$. Let $O=\bigcup \mathcal{V}$ and consider the disjoint closed sets $F_{i} \backslash O$ and $F_{j} \backslash O$. Since $X$ is normal we may assume that $d_{\alpha}\left(F_{i} \backslash O, F_{j} \backslash O\right)>0$. Moreover, let $d_{\alpha}$ be bounded by 1 . Define. now the admissible pseudo-metric $\rho_{m+1}$ on $X$ by

$$
\rho_{m+1}(x, y)=\max \left\{\rho_{m}(x, y)\right\} \cup\left\{\frac{1}{i \cdot j} d_{\alpha}(x, y) \mid \alpha=(i, j) \in A\right\} .
$$

If we put

$$
\rho(x, y)=\max \left\{\rho_{m}(x, y) / m \mid m \in \mathbf{N}\right\} \quad \text { for } x, y \in X,
$$

then it is obvious that

$$
\tau_{\rho_{1}} \subset \tau_{\rho_{2}} \subset \cdots \subset \tau_{\rho_{m}} \subset \cdots \subset \tau_{\rho} \subset 0
$$

where $\mathcal{O}$ is the collection of open subsets of $X$.

Define the equivalence relation $\sim$ on $\underset{\tilde{X}}{X}$ by $x \sim y \Leftrightarrow \rho(x, y)=0$. Let $\tilde{X}$ be the quotient space $X / \sim$ and let $q: X \rightarrow \tilde{X}$ be the decomposition mapping. Make a metric space of $\tilde{X}$ by putting $\tilde{\rho}(q(x), q(y))=\rho(x, y)$ for $x, y \in X$. Since $X$ is compact and $\rho$ is admissible, $q$ is a closed mapping and $(X, \tilde{\rho})$ is a compact metric space. If $x, y \in F_{1}$ such that $q(x)=q(y)$ then $\rho(x, y)=0$ and hence $\rho_{1}(x, y)=0$. Consequently, $f(x)=f(y)$ and since $q$ is closed there is a continuous $\tilde{f}: q\left(F_{1}\right) \rightarrow S^{n}$ with $\tilde{f} \circ q \mid F_{1}=f$. Obviously, $\left\{q\left(F_{i}\right) \mid i \in \mathbf{N}\right\}$ is a closed covering of $\tilde{X}$.

It remains to show that $\operatorname{dim}\left(q\left(F_{i}\right) \cap\left(F_{i}\right)\right)<n$ for $i \neq j$. Let $\mathcal{U}$ be a finite open covering of $q\left(F_{i}\right) \cap q\left(F_{j}\right)$ in $\tilde{X}$. We have that

$$
F_{i} \cap F_{j} \subset \bigcup q^{-1}[\mathcal{U}]=\bigcup\left\{q^{-1}(U) \mid U \in \mathcal{U}\right\} .
$$

Since $F_{i} \cap F_{j}$ is compact and since $q^{-1}[\mathcal{U}] \subset \tau_{\rho}$ there is a Lebesgue number $1 / m$ for $q^{-1}[\mathcal{U}]$, i.e. for every $x \in F_{i} \cap F_{j}, S_{1 / m}^{\rho}(x)$ is contained in some element of $q^{-1}[\mathcal{U}]$. Since $\rho_{1} \leq \rho_{2} \leq \rho_{3} \leq \cdots$ and every $\rho_{k}$ is bounded by 1 we have that

$$
S_{1 / m}^{\rho_{m}}(x) \subset S_{1 / m}^{\rho}(x) \text { for every } x \in X .
$$

Now there is a pseudo-metric $d$ on $X$ and a finite subcollection $\mathcal{V}$ of $\tau_{d}$ such that $d /(i \cdot j) \leq \rho_{m+1}, \operatorname{mesh}_{\rho_{m}} \mathcal{V}<1 / m$, ord $\mathcal{V} \leq n$ and $d\left(F_{i} \backslash O, F_{j} \backslash O\right)>0$, where $O=\bigcup \mathcal{V}$. Every element $V$ of $\mathcal{V}$ is in $\tau_{\rho}$ and hence $q^{-1}(q(V))=V$. This implies that $q[\mathcal{V}]=\{q(V) \mid V \in \mathcal{V}\}$ is an open collection in $\tilde{X}$ and has the property $\operatorname{ord} q[\mathcal{V}] \leq$ ord $\mathcal{V} \leq n$. We may assume that every member of $\mathcal{V}$ has points in common with $F_{i} \cap F_{j}$. It follows that for every $V \in \mathcal{V}$ there is a $U \in \mathcal{U}$ and an 
$x \in F_{i} \cap F_{j}$ such that $V \subset S_{1 / m}^{\rho_{m}}(x) \subset q^{-1}(U)$ and hence $q(V) \subset U$. In order to prove that $q\left(F_{i}\right) \cap q\left(F_{j}\right) \subset \bigcup \mathcal{V}$ select a $y \in F_{i}$ and a $y^{\prime} \in F_{j}$ such that $q(y)=q\left(y^{\prime}\right)$. Suppose that $y \notin O$. Since $O \in \tau_{\rho}, q^{-1}(q(O))=O$ and hence $y^{\prime} \notin O$. Then

$$
\begin{aligned}
\rho\left(y, y^{\prime}\right) & \geq \frac{1}{m+1} \rho_{m+1}\left(y, y^{\prime}\right) \geq \frac{1}{(m+1) \cdot i \cdot j} d\left(y, y^{\prime}\right) \\
& \geq \frac{1}{(m+1) \cdot i \cdot j} d\left(F_{i} \backslash O, F_{j} \backslash O\right)>0
\end{aligned}
$$

which contradicts $q(y)=q\left(y^{\prime}\right)$. This implies that $y \in O$ and $q(y) \in \bigcup q[\mathcal{V}]$. So we have proved that every finite open covering of $q\left(F_{i}\right) \cap q\left(F_{j}\right)$ can be refined by a finite open covering of order $\leq n$. This means that $\operatorname{dim}\left(q\left(F_{i}\right) \cap q\left(F_{j}\right)\right)<n$.

We have arrived at a position where we can apply Lemma 3 , yielding the existence of a continuous $g: \tilde{X} \rightarrow S^{n}$ that extends $\tilde{f}$. Then $g \circ q$ is the required extension of $f$.

REMARKS. We mention two applications of the theorem. The space $\mathbf{R}^{n}$ cannot be written as union of a sequence $F_{1}, F_{2}, F_{3}, \ldots$ of compact sets with $\operatorname{dim}\left(F_{i} \cap F_{j}\right)<$ $n-1$ for $i \neq j$. This follows directly from the fact that the identity mapping $\partial I^{n} \rightarrow \partial I^{n}$ is not extendable over $I^{n}$.

The second application concerns boundary sets in the Hilbert cube $Q$. If $\left(F_{i}\right)_{i=1}^{\infty}$ is a sequence of closed subsets of $Q$ such that $Q \backslash \bigcup_{i=1}^{\infty} F_{i}$ is homeomorphic to the Hilbert space $l^{2}$, then there is for each $n \in \mathbf{N}$ an infinite set $\left\{i_{m} \mid m \in \mathbf{N}\right\} \subset \mathbf{N}$ such that $\operatorname{dim}\left(F_{i_{m}} \cap F_{i_{m+1}}\right)>n$ for every $m \in \mathbf{N}$. This result was established in the author's thesis $[\mathbf{1}, 5.4 .6]$, where the theorem plays a key role.

\section{REFERENCES}

1. J. J. Dijkstra, Fake topological Hilbert spaces and characterizations of dimension in terms of negligibility, Thesis, University of Amsterdam, 1983; Math. Centre Tracts (to appear).

2. R. Engelking, Dimension theory, PWN, Warsaw, 1978.

3. __ General topology, PWN, Warsaw, 1977.

4. W. Sierpiński, Un théorème sur les continus, Tôhoku Math. J. 13 (1918), 300--303.

Mathematisch InstituUt, Universiteit Van Amsterdam, Roetersstraat 15, AMSTERDAM, THE NETHERLANDS 